\title{
Introduction
}

\section{Disease Markers: Molecular Biology of PTSD}

\author{
Rachel Yehuda \\ James J. Peters Veterans Affairs Medical Center, Bronx, NY, USA \\ E-mail: Rachel.Yehuda@va.gov
}

There have been significant advances in understanding the phenomenology and biology of PTSD in the more than thirty years since the disorder first appeared in the DSM-III. However, as we stand in the crossroads towards DSM-V, it is clear that scientific efforts in the examination of PTSD have yielded more questions than they have answered. PTSD was initially described as a condition in which "the essential feature is the development of characteristic symptoms following a psychologically traumatic event that is generally outside the range of usual human experience" (APA, 1980). It was not only implied, but made explicit in the DSM-III, that the symptoms result from the trauma exposure. Thus, the one question that investigators did not feel they had to attend to in the early years of research on this disorder was what causes PTSD. Rather, initial investigations focused on demonstrating that persons who experience extreme trauma showed alterations in biological systems that have also been implicated in chronic stress and/or identified as correlates of fear.

A variety of converging findings have now provided a scientific mandate to answer the question of what causes PTSD. First and foremost are results from epidemiologic studies demonstrating that only a proportion of trauma exposed persons develop PTSD. These studies have led to the conclusion that trauma exposure alone is not a sufficient causal agent. Furthermore, it has become clear that the findings related to stress or fear neurobiology may not be fully or completely applicable to the PTSD phenotype. It is true that the two major stress-responsive biological systems - the hypothalamic pituitary adrenal (HPA) axis and sympathetic nervous system (SNS) - seem to be dysregulated in PTSD. However, it has not been possible to conclude from extant findings that the PTSD neurobiology is analogous to the classical neurobiology of chronic stress or fear. While stress neurobiology arguably explains the emergence of some symptoms in the acute aftermath of trauma, it has not been able to explain either the persistence of those symptoms in the subset of trauma survivors who do not recover, or why such persons do not undergo what is arguably the defining hallmark of the stress response - the recalibration of biological systems towards homeostasis. Given these existing questions, the next generation of biological studies in PTSD must evaluate a broader range of biological mechanisms, and this examination must include molecular biology.

It is for this reason that we have compiled a volume that puts together a series of conceptually related papers on the emerging molecular biology of PTSD. In that this is a new field, a volume that summarizes various methodological and theoretical approaches may have value in creating a scaffolding for future studies.

Molecular biology is an approach that focuses on integrating information about the functioning of different cellular components. This includes how information travels from DNA to RNA, from RNA to protein synthesis, and ultimately from protein synthesis to function and behavior. Molecular biology is not only interested in the regulation of transcription and translation, but also in other processes of gene regulation including epigenetic regulation or genomic imprinting. The latter mechanisms are particularly interesting to examine in PTSD because they may reflect enduring consequences of environmental perturbations. In turn, epigenetic alterations can influence other molecular processes such as transcriptional and post-transcriptional regulation (e.g., sequestration, alternative splicing, trans- 
lational regulation, and both reversible and irreversible post-translational regulation). Whereas the field of psychiatry has begun to make use of molecular biology by identifying genetic association with symptoms, it has become clear that studies of genotype must be supplemented by efforts to evaluate the proteome, transcriptome and epigenetic aspects regulating cell function.

The ideal methodology for identifying a molecular biology of PTSD involves the development and testing of models that integrate genetic, epigenetic, neuroendocrine, and risk and resilience factors together with environmental factors to predict the development of PTSD. Such an approach would also account for contributing factors, would preferably be prospective (i.e., initiated even before trauma exposure) and longitudinal, and would consider sampling biases. Given the massive number of existing biological measures, this vision seems overwhelming if not prohibitively expensive. However, the papers in this volume take a step towards realizing this ultimate vision.

In the first paper, Yehuda and colleagues ask the question of why genes might be involved in the biology of PTSD at all. Of all psychiatric disorders, PTSD represents the quintessentially environmental condition because it cannot exist without the exposure. However, genotype may be important to PTSD in at least two major ways. First, the presence of a specific gene variant could increase the risk to environmental exposure. Second - and the more classical explanation for the role of genotype in PTSD - the presence of a specific gene variant can contribute to individual differences in response to an event. Indeed the important question that arises regarding gene and environment is whether, in fact, they areindependent. If genotype contributes to trauma exposure, than some effects that are interpreted as gene $\mathrm{x}$ environment interactions may simply be gene $x$ environment correlations. A true gene $\mathrm{x}$ environment interaction implies that environmental events are random, but exposure to trauma may not be independent of genotype. The authors also attempt to distinguish between an environmental exposure that interacts with a gene to produce a specific response, and one that modifies the gene (e.g., via epigenetic mechanisms). Importantly, epigenetic mechanisms can control the ways genes function because they regulate how information carried in the genotype is accessed. Thus functional changes in gene expression can theoretically occur independent of specific allelic variation. If this is true in practice, then the field of molecular biology of PTSD may begin to rely less on relatively static inherited genotype differences to explain why some people develop PTSD following trauma exposure and others do not.

Inarguably, the study of epigenetics is an important frontier in the molecular biology of PTSD. Schmidt and colleagues provide an elegant summary of the epigenetic mechanisms that may be of high relevance to PTSD, while also summarizing existing data from animal models and emerging clinical studies that support a role for these mechanisms in PTSD. The rationale for examining the epigenome in PTSD is to gain insight into the mechanisms that mediate communication between the environment and genes. To address the issue of enduring alterations in the HPA and SNS - that is, those that cannot be explained by current stress theory Schmidt et al. distinguish between effects of a traumatic "wound" rather than an ongoing challenge (i.e., "stressor"). The authors also examine the distinction between epigenetic processes that control the chromatin and those that alter the DNA molecule itself. Both changes modify access to biological information. Relevant modifications of chromatin can be accomplished by methylation and phosphorylation of histone proteins at defined sites, and also more rare modifications of histones, such as sumolyation, ubiquitination, ADPribosylation, biotinylation, carbonylation, deimination, proline isomerization, and glycosylation. These serve to explain how the DNA molecule becomes available for methylation and acetylation of bases which then directly alter gene expression generally by silencing the gene. The truly exciting idea presented by Schmidt et al. is that PTSD prophylaxis may come from secondary prevention using epigenetic therapy in PTSD during the incubation phase immediately following trauma exposure. Some of the novel approaches towards PTSD prophylaxis, such as treatment with high doses of glucocorticoids $[1,2]$, may be effective because they may interrupt the trajectory of symptom development that is the result of a post-traumatic epigenetic process.

The next section of this volume is devoted to clinical studies examining various aspects of the molecular biology in PTSD populations. The first of these studies by Amstadter and colleagues is an example of a classic candidate gene (and gene $\mathrm{x}$ environment) approach. Candidate genes are chosen for study based on their functional significance to a biological correlate of PTSD. In the past, the existence of a functional gene variant generally provided the justification for further examining more downstream molecular biological mechanisms leading to the phenotype. The challenge of this approach is to select a biological alteration that is highly specific to a disorder or symptom. In 
this study the authors have chosen to examine variation in the CRHR1 gene. This gene, and putative downstream CRH alteration, has been generally implicated in mood and anxiety disorder, but less specifically in PTSD. Rather, the alterations in the HPA axis that have been observed in PTSD appear to occur more at the level of the glucocorticoid receptor than at the level of hypothalamic CRH regulation per se. Nonetheless, in that variation in the CRHR1 gene was found to interact with a history of childhood maltreatment in another sample, it is interesting to consider whether this genotype might also interact with childhood pediatric injury. Even in this relatively small sample, an association between the CRHR1 gene variant and injury in the prediction of PTSD in children was observed. However, it is not clear to what extent this genotype might have also interacted with injury to predict other psychiatric symptoms of mood and depression. The implications of the findings may be substantial. To the extent that childhood maltreatment and pediatric injury are distinct events, then the gene $\mathrm{x}$ childhood trauma interaction observed in previous studies may reflect a true interaction, not merely a correlation. On the other hand, pediatric injury, like childhood maltreatment, may not occur in a vacuum, particularly in an inner city sample. This paper, then illustrates that much of molecular biological studies in PTSD will need to be interpreted and evaluated in the context of the sample chosen for study.

The above study provides a nice segue to the contribution of Sarapas et al. which uses a completely different strategy. Rather than a candidate gene approach, this study provides a genome-wide examination, which is hypothesis-generating. Instead of examining genotype, this study utilized microarray analysis to provide an unbiased survey of the mRNA expression level of nearly the entire human genome, allowing the identification of molecular pathways that have not yet been identified in PTSD. The assessment of gene expression changes can help identify a broader range of relevant pathophysiologic and risk factors in PTSD including those associated with epigenetics, gene transcription and protein synthesis. Of course when departing from studies of DNA, it becomes critical to consider the nature of the tissue that is being investigated. In the following three studies, gene expression and other analyses are performed in mononuclear leukocytes (which may or may not be models of the brain). The genes identified can then be genotyped to determine whether changes in RNA are functional expressions of inherited allelic variation (rather than resulting from environ- mental perturbations, eg. trauma exposure). Another advantage of the approach by Sarapas et al. is that the sample utilized was one in which risk for PTSD was not confounded with risk for trauma exposure. The sample studied was selected from a longitudinal and prospective epidemiological cohort of persons exposed to the 9/11 World Trade Center attacks who either had PTSD for at least two out of four longitudinal assessments or never developed PTSD following this event. Because some of the participants had also recovered from PTSD, the authors were able to identify genes associated with the development of PTSD, recovery from PTSD (i.e., "resilience genes"), and PTSD state symptomatology. That at least one of these genes, FKBP5 was related to glucocorticoid receptor sensitivity and was associated with cortisol levels suggests the beginning of identifying a functional molecular aspect of PTSD pathophysiology.

The contribution by Uddin et al. provides a very innovative template for future molecular biological studies. Here the authors used the existing literature to select genes that had been differentially expressed in other studies of PTSD (such as the aforementioned study), and examined those genes for changes in methylation. This approach is so interesting because it blends some of the unique opportunities of genome-wide examination with the discipline of a candidate gene approach. The authors asked whether the changes in gene expression are underpinned by an epigenetic mechanism (i.e., cytosine methylation). Since the literature on the molecular biology is relatively small, a manageable number of 33 genes were evaluated. Methylation microarray specifically focused on whether any of these candidate genes showed epigenetic signatures indicative of increased risk for PTSD. The authors further added a gene $\mathrm{x}$ environment analysis. Of the 33 candidate genes, only one was found to be associated with a higher methylation $\mathrm{x}$ cumulative trauma burden PTSD risk. It is difficult to draw too many conclusions about epigenetic changes from this study. The identified gene, MAN2Cl, has been implicated in t-cell functioning and is known to be involved in cancer progression. Thus there is no apparent reason why this gene should be differentially methylated in association with PTSD. Furthermore, the sample is relatively small and mixed with respect to trauma exposure. Yet the possibilities of using this type of approach are very important to think about. Indeed, one of the very challenging aspects of this kind of work is the number of variables (i.e., genes) when departing from a candidate gene approach. Here, a rational way of selecting genes for fur- 
ther examination was identified. Whereas typically a genome wide microarray analysis identifies genes that can be genotyped, in PTSD, the rationale for examining other molecular aspects is even more compelling (and does not rule out gene analysis).

Another very interesting approach to elucidate the molecular biology of PTSD is presented by O'Donovon et al. These authors also performed a gene expression study but sought to identify transcription factor binding motifs in promoter regions of differentially expressed genes from persons with PTSD and matched controls. Promotor regions are locations on the genes that are most likely to be subject to epigenetic modifications. This study is similar to the approach of Uddin et al., in that genes that have been demonstrated to show differential monocyte gene expression in PTSD were further examined to determine whether the expression changes stemmed from differences in upstream inflammatory, adrenergice or glucocorticoid transcriptional activity in the promoter DNA sequences. Like the other papers in this volume, this contribution also highlights the utility of analyzing gene expression at multiple levels to obtain insight into many different molecular aspects of PTSD. The authors chose to focus on inflammatory activity because this may be an important pathway that relates not only to the PTSD phenotype, but to the increased risk for physical disease associated with the disorder. One of the most fascinating aspects of this study is the very substantial gender difference that was observed. Men and women with PTSD both showed upregulation of target genes associated with the NF- $\kappa \mathrm{B} /$ Rel family of transcription factors, which convey inflammatory signals and down-regulation of target genes for the glucocorticoid receptor. However, men showed an upregulation of target genes for CREB/ATF transcription factors, which convey adrenergic signals from the SNS, while women showed a downregulation of these target genes. These findings may ultimately yield insight into gender differences in PTSD and should be examined further.

Flory et al. demonstrate how behavioral observations can implicate and be informative of molecular biological mechanisms even when molecular biological tools are not directly utilized. Here a careful examination of behavior, phenotype, and patterns of familial transmission, including attention to paternal vs. maternal associations, identified behaviors that are possibly underpinned by early developmental programming. In one sense, the question being asked in this paper is a simple one: Do adult children of Holocaust survivors report more emotional and physical health problems than comparable Jewish adults whose parents did not have any exposure to the Holocaust? The analysis performed demonstrated that Holocaust offspring did report having poorer emotional and physical health, but that these observations were preferentially associated with maternal, not paternal, Holocaust exposure. The subjective impression of poorer health was substantiated by the findings of greater usage of current psychotropic and other medicine for the treatment of medical disorders (such as for hypertension). Because of previously demonstrated links between maternal stress during pregnancy and the subsequent development of hypertension in adult offspring, it is suggested that the findings may be mediated by changes in early glucocorticoid programming. This mechanism has already been invoked as the molecular mechanism through which other observations, such as underlying lower cortisol levels and increased prevalence of PTSD in adult Holocaust offspring [2,3]. The paper is included to help expand the field of molecular biology to careful clinical observations.

The last paper in this volume is a review of gender differences in animal models of PTSD, which is particularly pertinent given the evidence for gender differences obtained by O'Donovon et al. The paper at the end of the volume rather than at the beginning is meant to demonstrate the uniqueness of research in PTSD where animal modeling has truly followed clinical observation. Indeed, animal studies have the potential of addressing many of the questions that have arisen in clinical studies, including those in this volume. These concern the relevance of monocytes as models of gene expression in the brain as well as issues related to gender differences. Indeed, the literature suggests that in PTSD animal models, gender differences are very strong, mirroring the observation of gender differences in the prevalence, but not necessarily the biology of PTSD. Animal models may also one day be used to explain some of the findings that relate specifically to transgenerational transmission of PTSD risk from pregnant mothers to offspring. An important idea that is presented is that prevalence differences do not actually speak to molecular or biological mechanisms associated with gender differences in vulnerability and resilience, and in fact, there are starkly contrasting findings between the human and animal data in this regard. These serve to heighten our interest and enthusiasm for future studies that identify relevant biological templates of individual variation and potential cultural (i.e., environmental and enduring molecular) influences. 


\section{References}

[1] G. Schelling, B. Roozendaal and D.J. De Quervain, Can posttraumatic stress disorder be prevented with glucocorticoids? Ann N Y Acad Sci 1032 (2004), 158-166.

[2] J. Zohar, R. Sonnino, A. Juven-Wetzler and H. Cohen, Can posttraumatic stress disorder be prevented? CNS Spectrum 14(1 Suppl 1) (2009), 44-51.
[3] R. Yehuda, A. Bell, L. Bierer and J. Schmeidler, Maternal, not paternal, PTSD is related to increased risk for PTSD in offspring of Holocaust survivors, J Psychiatr Res 42(13) (2008), 11041111.

[4] R. Yehuda, M.H. Teicher, J.R. Seckl, R.A. Grossman, A. Morris and L.M. Bierer, Parental posttraumatic stress disorder as a vulnerability factor for low cortisol trait in offspring of holocaust survivors, Arch Gen Psychiatry 64(9) (2007), 1040-1048. 


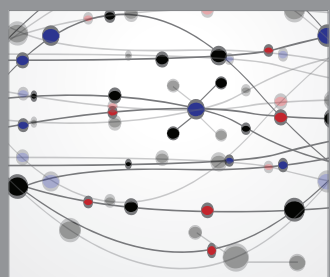

The Scientific World Journal
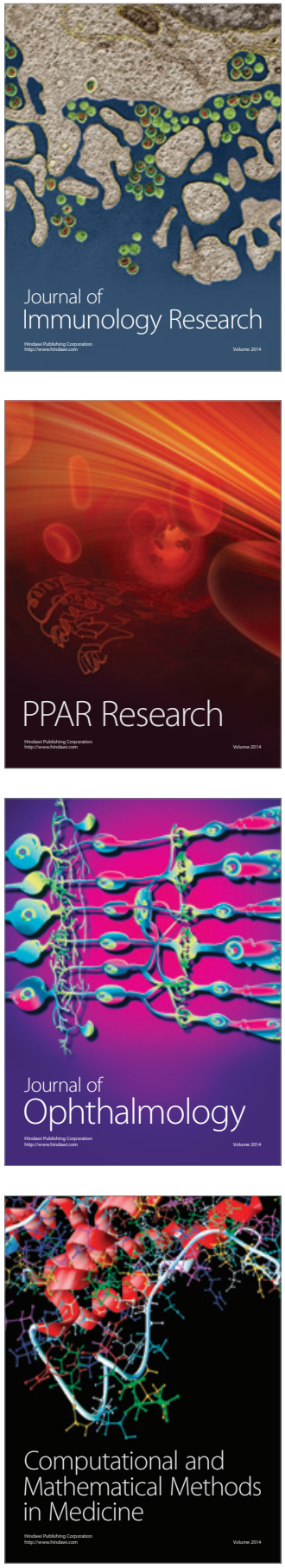

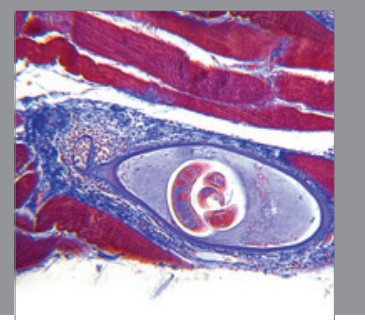

Gastroenterology

Research and Practice
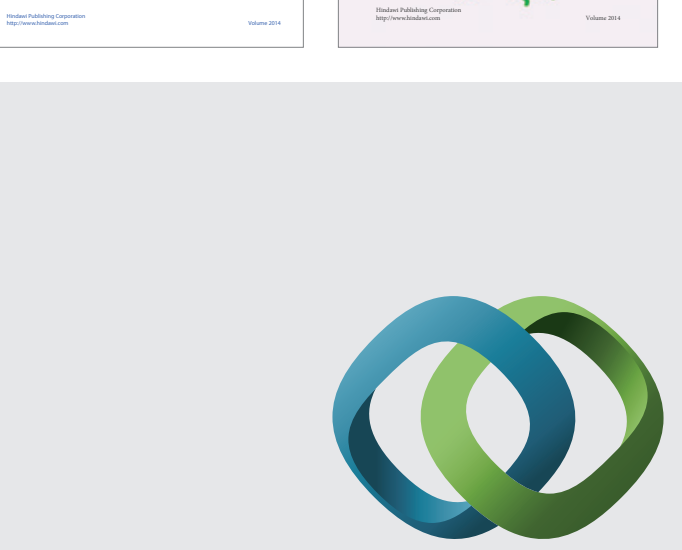

\section{Hindawi}

Submit your manuscripts at

http://www.hindawi.com
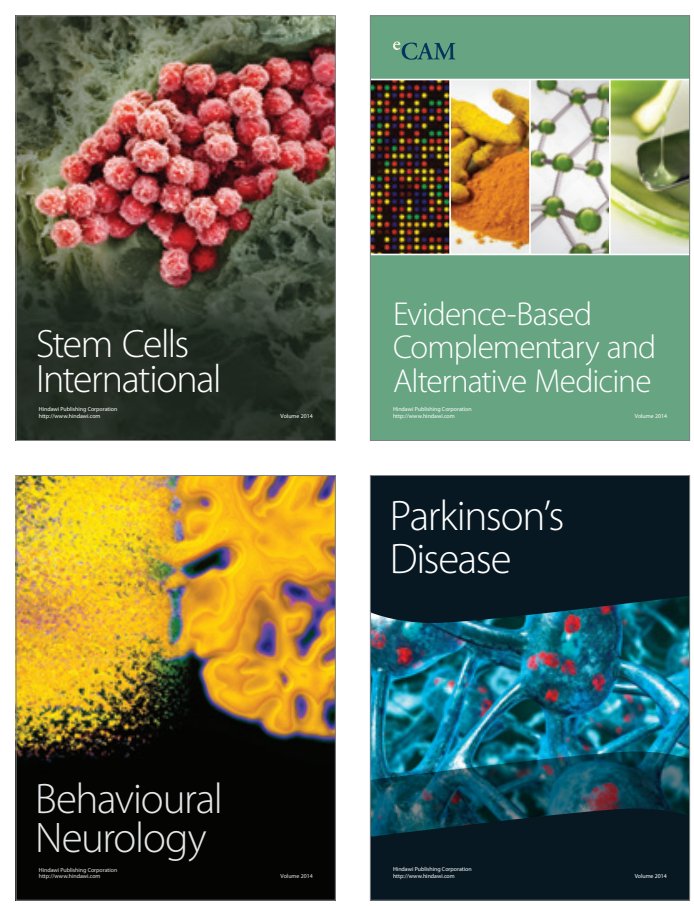

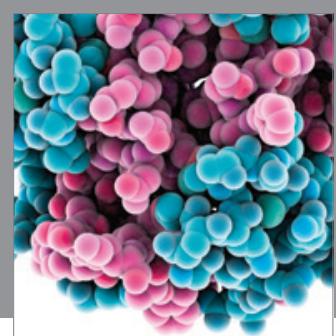

Journal of
Diabetes Research

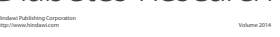

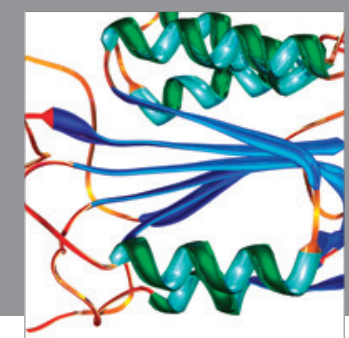

Disease Markers
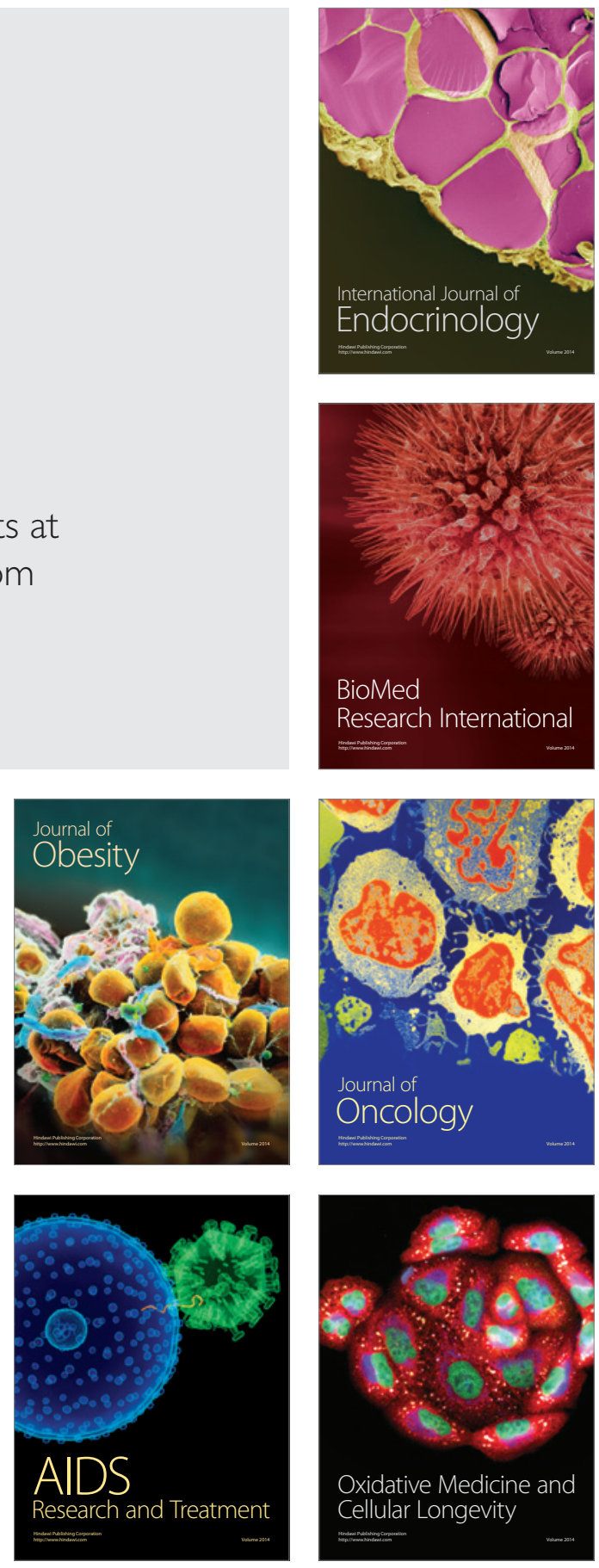